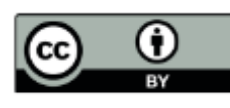

\title{
Acceso a servicios de salud: estudio de hogares en Palmira, Valle, Colombia, 2017*
}

\section{Access to health services: Household Study in Palmira, Valle, Colombia 2017}

\section{Acesso aos serviços de saúde: estudo das famílias em Palmira, Valle, Colômbia, 2017}

Recibido: 05 de noviembre de 2019. Aceptado: 10 de noviembre de 2020. Publicado: 30 de junio de 2021.

DOI: https://doi.org/10.11144/Javeriana.rgps20.asse

\author{
Clara Inés Sánchez Perafán \\ Pontificia Universidad Javeriana, Colombia \\ ORCID: https://orcid.org/0000-0002-5974-4130 \\ Diana Liceth Mora Rojas \\ Pontificia Universidad Javeriana, Colombia \\ ORCID: https://orcid.org/0000-0003-4848-4457 \\ Víctor Manuel Botero Henao \\ Pontificia Universidad Javeriana, Colombia \\ ORCID: https://orcid.org/0000-0002-7447-7552 \\ Victoria Eugenia Estrada ${ }^{a}$ \\ Pontificia Universidad Javeriana, Colombia \\ ORCID: https://orcid.org/0000-0002-4905-8671
}

Para citar este artículo: Sánchez CI, Mora DL, Botero VM, Estrada VE. Acceso a servicios de salud: estudio de hogares en Palmira, Valle, Colombia, 2017. Rev Gerenc Polit Salud. 2021;20. https://doi.org/10.11144/Javerian a.rgps20.asse

\footnotetext{
* Artículo de investigación

a Autora de correspondencia. Correo electrónico: veestrada@javerianacali.edu.co
} 


\section{Resumen}

Introducción. El acceso a servicios de salud es una de las principales preocupaciones de las políticas sanitarias. En el Valle del Cauca es escasa la información local sobre acceso efectivo desde la perspectiva de la población. Objetivo. Analizar el comportamiento del acceso a los servicios de salud de la población urbana del municipio de Palmira, Valle del Cauca en el año 2017. Método. Estudio cuantitativo transversal, a través de encuesta aplicada a una muestra de 242 hogares; el muestreo fue probabilístico, estratificado, multietápico por conglomerados. Resultado. Urgencias y consulta médica evidencian alta resolución de la necesidad demandada y satisfacción, pero baja oportunidad; la continuidad de la atención se ve afectada por excesiva tramitología, no autorización de servicios y no entrega de medicamentos; en consulta especializada la oportunidad es baja; hospitalización reportó mejor en la oportunidad, alta satisfacción y alta resolución de la necesidad; en servicios preventivos la escasa promoción de la oferta determina el poco uso de los servicios, y en varios casos, es mayor el uso que la promoción. Discusión. Se corroboran resultados de estudios anteriores; se destaca la importancia de las barreras administrativas y estructurales del sistema para comprender la problemática del acceso en Colombia. Conclusiones. Pese al aumento en el aseguramiento de la población persisten problemas que impiden el acceso real de la población a los servicios de salud, relacionados con oportunidad, continuidad, complementariedad y gasto de bolsillo; la escasa promoción de los servicios preventivos evidencia el enfoque curativo del modelo de atención en salud en Colombia.

Palabras clave: Accesibilidad a los servicios de salud, servicios preventivos de salud, calidad de la atención de salud.

\section{Abstract}

Introduction. Access to health services is one of the principal health policy concerns. In Valle del Cauca, local information on effective access from the perspective of the population is scarce. Objective. Analyze the behavior of access to health services in the Palmira, Valle del Cauca urban population in 2017. Method. Cross-sectional quantitative study, through a survey applied to a sample of 242 households; the sampling was probabilistic, stratified, multistage by conglomerates. Results. Emergencies and medical consultation show a high resolution of the demanded need and satisfaction, but low opportunity; continuity of care is affected by excessive paperwork, non- authorization of services, and non-delivery of medicine. For specialized consultation, the opportunity is low. Hospitalization reported better opportunity, high satisfaction and high resolution of needs. In preventive services, the lack of promotion of the services determines their scarce use; in several cases, the use is higher than the promotion. Discussion. The results of previous studies were corroborated. The importance of the administrative and structural barriers of the system is highlighted in order to understand the problem of access in Colombia. Conclusion. Despite the increase in the population's insurance coverage, problems persist that prevent real access of the population to health services, related to opportunity, continuity, complementarity and out-of-pocket expenses; the scarce promotion of preventive services demonstrates the curative focus of the health attention model in Colombia.

Keywords: Accessibility to health services, preventive health services, quality of health care.

\section{Resumo}

Introdução. O acesso aos serviços de saúde é uma das principais preocupações das políticas de saúde; no Valle del Cauca, as informações locais sobre o acesso efetivo do ponto de vista da população são escassas. Objetivo. Analisar o comportamento do acesso aos serviços de saúde da população urbana do município de Palmira, Valle del Cauca em 2017. Método. Estudo transversal quantitativo, por meio de inquérito aplicado a uma amostra de 242 lares; a amostragem foi probabilística, estratificada, multietápica por conglomerados. Resultado. Emergências e consulta médica mostram alta resolução da necessidade demandada e satisfação, mas baixa oportunidade; a continuidade do atendimento é afetada por excesso de burocracia, não autorização de serviços e não entrega de medicamentos; na consulta especializada, a oportunidade é baixa; internação relatou melhor oportunidade, alta satisfação e alta resolução da necessidade; nos serviços preventivos, a escassa promoção da oferta determina a escassa utilização dos serviços, sendo em vários casos, a utilização superior à promoção. Discussão. Resultados de estudos anteriores são corroborados; destaca-se a importância das barreiras administrativas e estruturais do sistema para entender o problema de acesso na Colômbia. Conclusões. Apesar do aumento da cobertura em saúde da população, persistem problemas que impedem o acesso real da população aos serviços de saúde, relacionados com a oportunidade, continuidade, complementaridade e despesas do próprio bolso; a escassa promoção dos serviços preventivos evidencia o enfoque curativo do modelo de atendimento à saúde na Colômbia.

Palavras-chave: Acessibilidade aos serviços de saúde, serviços preventivos de saúde, qualidade da assistência à saúde. 


\section{Introducción}

El acceso a los servicios de salud es uno de los principales temas de las políticas sanitarias que en términos operativos se ha definido como:

el funcionamiento en la puerta de entrada a los sistemas de salud sea cual fuere su naturaleza (pública, privada o mixta), así como la relación entre pacientes que demandan atención en salud y diferentes servicios que componen tales estructuras sanitarias (1 p.14).

La investigación sobre acceso a servicios de salud se soporta sobre distintos modelos conceptuales (2) entre los que destacan: la lógica de los mínimos decentes (3-5) relacionado con la definición de un paquete básico de servicios de un nivel específico de calidad, con lo cual los tomadores de decisiones definen un rango prioritario de servicios según los recursos financieros disponibles.

La lógica del mercado define el acceso a los servicios de salud en relación con la dinámica entre la oferta y la demanda, por lo que la equidad en el acceso -si bien no es un atributo visible del modelo- se vincula con la cobertura efectiva (6-8). Desde la lógica de los factores y la multicausalidad (9-12), el acceso a los servicios de salud es el resultante de factores de oferta como ubicación, disponibilidad y costo; y de factores de demanda tales como carga de enfermedad, conocimiento, actitudes, habilidades y autocuidado, la accesibilidad, aceptabilidad, disponibilidad y acomodación, asequibilidad y oportunidad. En este modelo el acceso equitativo está determinado por el comportamiento de factores individuales (conductas y creencias).

Desde la lógica de las necesidades, éstas son estimadas con base en indicadores de morbilidad, mortalidad y circunstancias socioeconómicas; y el acceso se define con base en patrones históricos de utilización de servicios de salud. Esta concepción ha sido ampliamente debatida pues se considera que estos modelos se basan en supuestos generales, como que los niveles de necesidad son los mismos en cada grupo estudiado. Al respecto, Goddard y Smith (13) proponen que el concepto operacional de necesidad se derive del análisis de cada grupo de población en relación con sus circunstancias de acceso, utilización, calidad y resultados de salud; en este sentido cobra importancia el análisis del acceso desde la población. En este modelo la equidad se entiende como "igual acceso para igual necesidad" e "igual utilización para igual necesidad".

Y finalmente, desde la lógica de la justicia social y el derecho a la salud, Braveman y Gruskin (14) plantean que el derecho a la salud debería constituir el marco para la organización de los sistemas de salud trascendiendo la perspectiva de los resultados en salud e involucrando los determinantes de la salud en los diferentes grupos poblacionales. En este modelo la equidad en el acceso se entiende en un sentido horizontal vinculada a la igualdad de oportunidades para acceder a la atención en salud, y en sentido vertical que implica acceso distinto para diferentes niveles de necesidad (2).

Frenk (8) plantea que la accesibilidad es producto de la relación entre los obstáculos en la búsqueda y la obtención de atención generados por la organización de los servicios de salud 
y las capacidades de la población para superar esos obstáculos, y que depende de los recursos disponibles en un contexto particular. El autor menciona tres dimensiones a partir de las cuales se relacionan los obstáculos del sistema con las capacidades de la población: (i) la ecológica, que relaciona los problemas que surgen por la ubicación de los lugares de atención con los recursos de tiempo y transporte de los usuarios; (ii) la financiera, que vincula los costos de la obtención de atención con la capacidad financiera de los usuarios; y (iii) la organizacional, relacionada con los tiempos de espera, la disponibilidad de tiempo libre y la tolerancia a las demoras por parte de los usuarios. La distancia, el costo o el tiempo de demora en la atención no dan cuenta por sí mismos del acceso sino que lo hacen al ponerlos en relación con el ingreso y el tiempo disponible de una población (1). El concepto "barreras en el acceso" se refiere tanto a estos elementos que impiden o dificultan recibir la atención una vez detectada la necesidad, como a los mecanismos a través de los cuales se dificulta la utilización de los servicios.

A partir de 1991 con el nuevo Sistema General de Seguridad Social en Salud, el gobierno colombiano logró una sobresaliente cobertura universal del aseguramiento de la población, sin embargo, es necesario tener en cuenta la diferencia entre los conceptos cobertura y acceso. Shengelia et al. (15) se refieren al acceso en tanto cobertura como la probabilidad de recibir la atención de salud necesaria y la disponibilidad de los recursos de salud; de esta manera amplía su dimensión e incluye factores geográficos, económicos y sociales. En Colombia el aumento de la cobertura fue el mecanismo a través del cual la Ley 100 de 1993 pretendió facilitar el acceso a la salud en el país. Esta situación es controvertida puesto que no representó un acceso real a los servicios de salud. Algunos ejemplos documentan esta situación: las múltiples quejas ante las entidades de control que demuestran fallas reiterativas en el actual sistema de salud, la pérdida de la autonomía de los médicos para ordenar apoyos diagnósticos y tratamientos efectivos, el incumplimiento de la portabilidad generando barreras en la atención médica y más grave aún la negación de los servicios lo que ha derivado en la interposición de acciones de tutelas para reclamar el derecho (16).

La ley estatutaria 1751 de 2015 intentó subsanar dichos problemas al contemplar, entre otras medidas, que los trámites administrativos no pueden estar a cargo de los usuarios, que la regulación de los precios de los medicamentos sea en toda la cadena y que la tutela en materia de salud no puede negarse bajo ninguna circunstancia (16). La implementación de guías de práctica clínica (GPC) busca fortalecer el acceso a la atención preventiva al establecer la obligatoriedad en la realización de acciones de detección temprana en patologías prioritarias según perfil epidemiológico con alcance limitado en su aplicación (17). Las brechas entre aseguramiento y acceso real son evidentes si se tiene en cuenta que mientras la cobertura de aseguramiento aumentó del 56,9\% al 90,8\% entre 1997 y 2012, el acceso a los servicios de salud, entendido como el porcentaje de personas que utilizó los servicios médicos al momento de necesitarlos, disminuyó en el mismo período al pasar de 79,1\% al 75,5\% $(18,19)$. Así, uno de los principales retos que conlleva el actual Sistema General de Seguridad Social en Salud (SGSSS) en el país es lograr el acceso efectivo a los servicios de salud. 
En la literatura existen importantes aportes sobre las diferencias en el acceso según régimen de afiliación, satisfacción de los usuarios especialmente en servicios curativos (20) e identificación de barreras y estrategias adoptadas por los usuarios para solventarlas (21). En el periodo 2000-2013, Tovar y Arrivillaga (1) exponen que de 31 artículos publicados la mayoría (32,2\%) trataba de los efectos de la Ley 100 de 1993 en el acceso a los servicios; un 22,6\%, en el acceso a servicios específicos (materno-infantil, urgencias, citología, servicios preventivos); otro 22,6\%, en el acceso de grupos vulnerables y un 19,35\% en la equidad entre géneros, etnia y el uso de servicios. Son más los estudios que privilegian el análisis del y desde el sistema, y menos aquellos que centran su atención en la perspectiva de la población y de necesidades particulares de grupos específicos (22-24).

Las autoridades sanitarias del departamento del Valle del Cauca han realizado esfuerzos dirigidos a minimizar los problemas de acceso y reducir las brechas de inequidad en los servicios de salud. Sin embargo, no se ha podido analizar el resultado de estos esfuerzos ya que no se cuenta con información local que muestre la situación de acceso efectivo a los servicios de salud desde la perspectiva de la población. La información existente se limita a aquella que históricamente se ha utilizado desde las instituciones para la medición de indicadores de coberturas de afiliación al sistema, disponibilidad de recurso humano en salud, disponibilidad de camas hospitalarias, la cual no permite analizar la relación con la demanda potencial y el uso de los servicios (25).

Para el 2016 en el Valle del Cauca la cobertura de aseguramiento al sistema fue de 92,2\%, que es más baja que la del nivel nacional (95,5\%), mientras que en Palmira esta cobertura fue de 99,2\% (26). Sin embargo, en relación con el acceso efectivo no se cuenta con información de fuente primaria y desde la perspectiva de la población. Lo anterior motivó la realización de este estudio, el cual se planteó como objetivo analizar el comportamiento del acceso a los servicios de salud de la población urbana del municipio de Palmira, Valle del Cauca, en el año 2017, con el ánimo de aportar elementos de análisis sobre la realidad del acceso a los servicios de salud desde la perspectiva de los hogares, que contribuya a la definición y redefinición de medidas, estrategias y/o políticas de mejoramiento del acceso, de acuerdo con las necesidades de la población.

\section{Materiales y métodos}

Tipo de estudio. Se realizó un estudio cuantitativo transversal a través de encuesta estructurada aplicada a las/los jefes de hogar de una muestra de 242 hogares de la zona urbana del municipio de Palmira, departamento Valle del Cauca, Colombia.

El diseño muestral. Diseño probabilístico, estratificado, multietápico por conglomerados (muestreo complejo), se conformaron cuatro estratos estadísticos correspondientes al nivel sociodemográfico de las personas (estrato de la vivienda donde residen). En cada estrato estadístico se aplicaron las tres etapas de muestreo: en la etapa I se seleccionan sectores cartográficos, dentro de estos las manzanas (etapa II), en la etapa III se seleccionan viviendas en las manzanas seleccionadas y en cada hogar se identifica el respondiente idóneo. La selección 
de unidades estadísticas en cada etapa se realiza por muestreo aleatorio simple. Para la selección de los hogares se realizó un reconocimiento cartográfico de la zona que permitió determinar que este correspondía a una vivienda y no a una fábrica o negocio, y sobre la base del plano de la ubicación de las viviendas, con el fin de garantizar la aleatoriedad de los mismos.

Marco de muestreo. El marco de muestreo disponible en el Centro Nacional de Consultoría lo constituye el listado de viviendas, hogares y personas (VIHOPE) del Censo Nacional de Población de 2012 realizado por el DANE. Esta herramienta permitió la selección de manzanas, viviendas y personas de la ciudad de Palmira y su cartografía.

Muestra: la estimación del tamaño de la muestra para la zona urbana del municipio de Palmira se calculó teniendo en cuenta los siguientes aspectos: una proporción de no uso de los servicios de salud de 50\%, un nivel de confianza del 95\%, un error de estimación del 5\% y un efecto del diseño de 2 (18). Con base en los aspectos anteriores y asumiendo una tasa de no respuesta del $20 \%$, el resultado de la estimación del tamaño muestral fue de 242 hogares.

Factores de expansión. En la investigación se consideró una estratificación estadística por nivel socioeconómico (NSE) del hogar, debido a que es probable que haya diferenciales en los resultados dependiendo del NSE al que pertenece el hogar: el nivel socioeconómico bajo (estratos 1 y 2) representa el 58\% de los hogares de Palmira, el nivel socioeconómico medio (estrato 3) representa el 28\% mientras que hogares de estratos altos (4-6) representan el restante $9 \%$. Como ejemplo se realizaron 78 encuestas en estrato 3 y el tamaño del universo del estrato 3 es de 22.212 hogares. Como resultado, el factor de expansión de un hogar de estrato 3 de Palmira es de 284,8 . Ver tabla 1.

Tabla 1. Universo, muestra y factor de expansión de hogares por estrato socio-económico

\begin{tabular}{|c|c|c|c|}
\hline Estrato & Muestra & Hogares & $\begin{array}{c}\text { Factor de } \\
\text { expansión }\end{array}$ \\
\hline 1,2 & 139 & 49.585 & 356,77 \\
\hline 3 & 78 & 22.212 & 284,77 \\
\hline $4,5,6$ & 25 & 7.289 & 291,56 \\
\hline Total & 242 & 79.086 & - \\
\hline
\end{tabular}

Fuente: elaboración propia. 
Diseño de la encuesta. La encuesta utilizada para este trabajo está conformada por 60 preguntas organizadas en cinco módulos que indagan por aspectos sociodemográficos del jefe del hogar, aspectos sociodemográficos del hogar, acceso a servicios preventivos, acceso a servicios curativos y gasto de bolsillo relacionado con el acceso. Esta es una adaptación de la Encuesta de Acceso a Servicios de Salud para hogares colombianos, diseñada y validada a través de un estudio piloto por Arrivillaga et al. (25).

Recolección de la información. Las encuestas fueron aplicadas de manera presencial por el centro nacional de consultoría y la información fue recolectada entre julio y agosto de 2017. Una vez identificados y seleccionados los hogares, los encuestadores, previamente capacitados llevaron a cabo la firma del consentimiento informado a todos los participantes y se aplicaron medidas para la garantía de confidencialidad de la información y, por último, se llevó a cabo la entrevista.

Análisis de la información. Se realizó un análisis descriptivo de las variables estableciendo frecuencias absolutas y relativas; se caracterizó el acceso de los servicios curativos básicos, preventivos y gasto de bolsillo utilizando comparación de promedios, diferencias y proporciones. El acceso a la atención preventiva se valoró a partir de dos componentes: la promoción de la oferta de servicios preventivos por parte de las EAPB y el uso de los servicios preventivos, por parte de las familias, para cada grupo de edad en el último año, teniendo en cuenta los programas existentes según etapa de ciclo vital, de acuerdo con la normatividad colombiana. La pregunta que permitió indagar el primer componente fue: “¿Su EPS le ha ofrecido a usted o a un miembro de su familia, en el último año...?”. La pregunta que permitió indagar el segundo componente fue: “¿Ha consultado o ha recibido atención, en el último año, usted o algún miembro de su familia por...?”.

\section{Resultados}

\section{Caracterización sociodemográfica de los participantes}

La distribución por sexo de los respondientes fue 50\% hombres y 50\% mujeres; $2 \%$ se reconoció como indígena y $18 \%$ como negro mulato o afro descendiente; $62 \%$ de los encuestados estaba casado y $22 \%$ soltero; $65 \%$ era mayor de 45 años, 2\% tenía entre 18 a 19 años; 42\% tenía educación secundaria, 33\% educación primaria y 2\% estudios de postgrado. La distribución por estrato socio-económico fue acorde a la cartografía, más del $80 \%$ pertenecía a los estratos socioeconómicos 2 y $3 ; 76 \%$ reportó ingresos entre 1 y 3,5 SMMLV y 20\% menos de 1 SMMLV; $28 \%$ trabaja como independiente, $22 \%$ es pensionado; cerca del $50 \%$ es trabajador remunerado y $18 \%$ trabaja sin remuneración. Un $46 \%$ vive en arriendo, $39 \%$ en casa propia y solo un $15 \%$ en vivienda familiar; $62 \%$ pertenecía al régimen contributivo y $36 \%$ al régimen subsidiado; en los estratos 1 y 2 el 55\% pertenece al régimen contributivo y $43 \%$ al subsidiado, y en los estratos 4 , 5 y 6 al 80\% régimen contributivo y 16\% al subsidiado; la mayoría afiliados a Emssanar (30\%), seguida por Nueva Eps (17\%), Coomeva (14\%), y con menor participación S.O.S, Salud Total, Comfenalco, Comfandi, Cafesalud y Sanitas. 
Clara Inés Sánchez Perafán / Diana Liceth Mora Rojas / Víctor Manuel Botero Henao / Victoria Eugenia Estrada

\section{Acceso a servicios curativos}

\section{Asistencia a servicio de urgencias - Estancia en el servicio de urgencias}

En los últimos 6 meses un 50\% de los hogares consultó el servicio de urgencias, en relación con la oportunidad en la atención, medida a través del tiempo de espera entre la llegada del paciente y la consulta médica, se encontró que un $25 \%$ de las personas se demoraron más de dos horas en ser atendidas; un 30\%, entre 31 minutos y 2 horas, un 19\%, 30 minutos; mientras que sólo un $23 \%$ fueron atendidas inmediatamente.

La satisfacción de las personas, como un elemento de la calidad de la atención en urgencias expresada en "sí recibió la atención necesaria frente a su problema de salud", reportó que el $75 \%$ resolvió su problema y el restante $25 \%$ no, de estos $41 \%$ refirió no haber sido atendido debido a que el caso era leve y fue remitido a consulta prioritaria, $39 \%$ desconoce las razones por las que no fue atendido y $16 \%$ desistió de la atención por excesivo tiempo de espera; $4 \%$ de las personas no recibió atención por no presentar el carnet, convirtiéndose esto en una barrera administrativa para el acceso. El 79\% consideró que la calidad de la atención fue buena o muy buena, $16 \%$ mala y $5 \%$ muy mala.

\section{Consulta por médico general}

Del $80 \%$ de los hogares que consultó al médico general en los últimos 6 meses, un 35\% fue atendido en menos de una semana; $37 \%$, entre 2 y 4 semanas y para un $11 \%$ la cita fue asignada de 1 a 3 meses, lo que indica incumplimiento de los estándares de oportunidad, la cual establece un máximo de 3 días. Desde la percepción de los usuarios, el 93\% considera que recibió la atención necesaria a su problema, en tanto un 3\% cubrió la atención con recursos propios o de sus familiares.

De aquellos que continuaban en tratamiento $(28 \%)$, un $12 \%$ indicó exceso de trámites para la obtención de los medicamentos, un $11 \%$ no recibió autorización, un $2 \%$ no contaba con los recursos para los copagos de los servicios adicionales y un $2 \%$ no tenía tiempo de hacer los trámites necesarios para continuar con la atención. En relación con el acceso a los medicamentos, el $82 \%$ de quienes asistieron a consulta recibió formulación de medicamentos, de ellos, al $72 \%$ le fue entregado el medicamento y al restante $28 \%$ no. Las razones de la no entrega fueron: un $21 \%$ porque no estaban incluidos en el POS, un $27 \%$ no realizó las gestiones para reclamarlos, un $15 \%$ acudió a médico particular, y para un $31 \%$ los medicamentos no estaban disponibles. Lo anterior evidencia dificultades en relación con la continuidad de la atención.

Las razones por las cuales las personas no consultaron al médico general (9\%) fueron: la cantidad de trámites requeridos para la cita (23\%), el mal servicio (15\%), no lo ha necesitado (36\%) y no confía en los médicos (11\%). Otras razones dadas fueron la falta de tiempo o la falta de dinero. 


\section{Consulta por especialista}

De los atendidos por medicina general, el 52\% fue remitido a un especialista; de ellos $13 \%$ al internista, $12 \%$ a ginecología, $12 \%$ a urólogo y $11 \%$ a oftalmología. La oportunidad se valoró a partir de dos parámetros: el tiempo entre la solicitud y la autorización de la cita; y el tiempo entre la autorización y la consulta. Con relación al primero, con un rango de 1 a 30 días, un 30\% esperó entre 2 a 4 semanas y un $27 \%$, menos de una semana. En cuanto al segundo, el $66 \%$ de los casos esperó entre 2 semanas y 3 meses, un $10 \%$ esperó entre 4 y 6 meses.

\section{Hospitalización}

El 17\% de los hogares reportó la necesidad de hospitalización en los últimos 6 meses, de ellos un $22 \%$ señaló tener un traslado inmediato a la cama asignada desde la orden de hospitalización, el $56 \%$ el mismo día y para $20 \%$ de los casos se tardaron entre 2 y 3 días. El $86 \%$ de los que requirieron hospitalización considera que le brindaron la atención necesaria para el problema de salud. El $72 \%$ percibe el servicio como muy bueno o bueno, y el restante $28 \%$ como malo o muy malo. Un $5 \%$ de los hogares utilizó recursos propios o de familiares para cubrir los gastos de la hospitalización.

\section{Gasto de bolsillo}

El 69\% de los hogares incurrió en gastos extras como transporte, alojamientos y alimentación, pagando en promedio $\$ 30.980$ pesos por la última atención recibida, el $52 \%$ pagó cuotas moderadoras o copagos en la última atención recibida con un promedio de $\$ 6,724$ pesos. El $50 \%$ realizó un pago por medicamentos, exámenes y procedimientos no autorizados por la EPS con un promedio de $\$ 24.277$ pesos, el 12\% pagó por lentes, audífonos o aparatos ortopédicos. El promedio de gastos fue de $\$ 200.202$ pesos, el $4 \%$ tuvo otro tipo de gastos, entre los que sobresalen papelería y fotocopias.

\section{Acceso a servicios preventivos}

Los resultados muestran que un $48 \%$ de los participantes consultó al médico sin estar enfermo: un $6 \%$ fue al odontólogo, y un $22 \%$ tanto al médico como al odontólogo; un $24 \%$ no solicitó consulta preventiva.

Promoción de la oferta y uso de servicios preventivos en niños menores de 1 añoPromoción de la oferta y uso de servicios preventivos en niños menores de 1 año

El control postparto y la valoración integral del recién nacido es un servicio que en mayor proporción fue prestado porque el usuario buscó el servicio y no por promoción de la oferta por parte de la EAPB. Las actividades más promocionadas fueron vacunación (100\%) valoración del recién nacido $(89 \%)$, valoración del niño sano $(89 \%)$ y control postparto $(78 \%)$; las menos 
promocionadas fueron las actividades educativas en puericultura (22\%) y la visita domiciliaria al recién nacido (22\%). Cabe resaltar que la vacunación es el único servicio que fue ofertado y utilizado en un $100 \%$, lo cual puede ser explicado por los esfuerzos de los actores de salud (EAPBs - Secretarias de Salud), en torno al cumplimiento de la política pública "cero barreras" en vacunación. Ver figura 1.

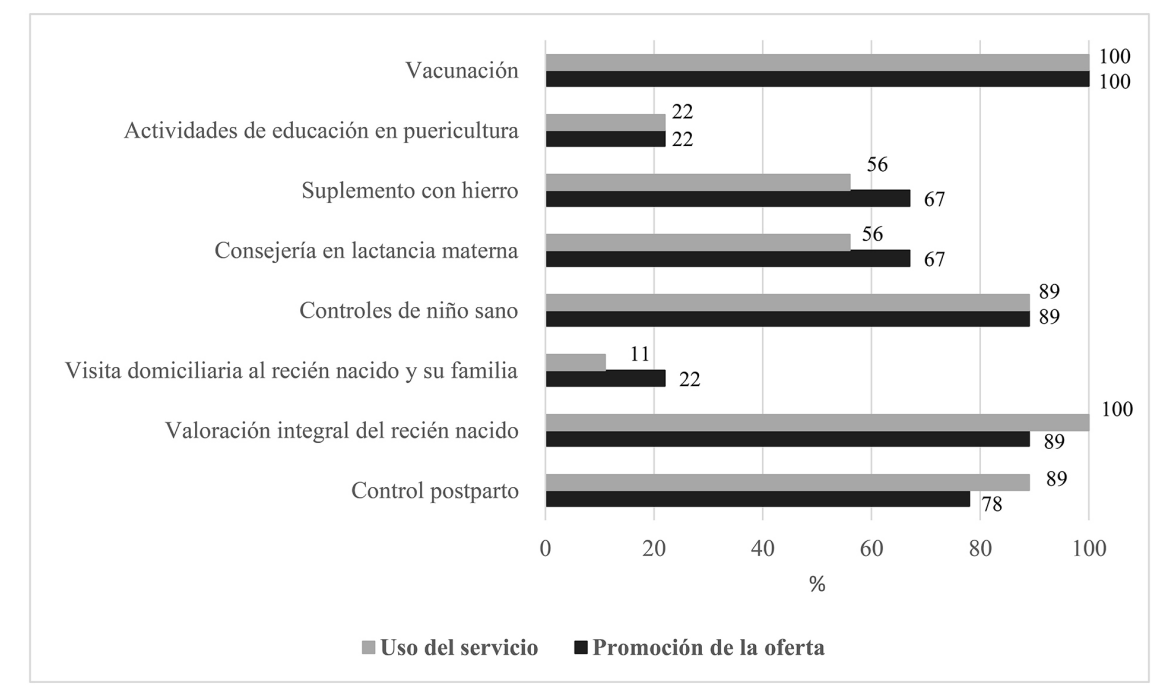

Figura 1. Promoción de la oferta y uso de servicios preventivos en niños menores de 1 año Fuente: elaboración propia.

\section{Promoción de la oferta y uso de servicios preventivos en niños entre 1 y 5 años}

Las actividades más promocionadas por las EAPB fueron vacunación (94\%), control del niño sano $(77 \%)$ y suministro de antiparasitarios $(75 \%)$. Vale resaltar que no se logra la meta del $100 \%$ indicada en las guías de práctica clínica (GPC). En correspondencia, las actividades más promocionadas son las que reportan un mayor uso y aquellas con menor promoción presentan bajo uso, evidenciando la importancia de esta actividad por parte de las EAPB, si se quiere lograr el incremento de las coberturas en actividades preventivas. Sin embargo, en todos los servicios (excepto en actividades de educación en el cuidado y suplemento con hierro) el uso de los mismos tuvo porcentajes superiores a su promoción.

\section{Promoción de la oferta y uso de servicios preventivos en niños entre 6 y 10 años}

Los servicios más promocionados en niños entre 6 a 10 años fueron: vacunación (79\%) y control del niño sano (76\%), suministro de antiparasitarios (72\%) y actividades de salud oral (67\%). Y con menos importancia la promoción de actividades educativas (42\%) y la valoración visual (45\%). En el caso de la valoración visual es necesario tener en cuenta que la Resolución 412 de 2007 estableció esta actividad a los 4, 11 y 16 años, lo que puede explicar en parte este resultado. 
En todos los servicios, excepto en educación, el uso de los mismos tuvo porcentajes superiores a su promoción. Desde el punto de vista de las GPC los porcentajes de utilización de servicios educativos, valoración visual y salud oral distan de manera importante del cumplimiento de las metas establecidas en la norma (8).

\section{Promoción de la oferta y uso de servicios preventivos en mujeres entre 11 y 19 años}

Las actividades más promocionadas en este grupo de edad fueron consulta por salud oral $(62 \%)$, agudeza visual (43\%), citología vaginal (29\%) y consulta médica preventiva (27\%). Los servicios con más baja promoción de la oferta y uso fueron consejería de planificación familiar $(25 \%)$ y consejería pre-concepcional $(15 \%)$. La consulta médica o psicológica por algún trastorno alimentario es una actividad que ni se oferta, ni se utiliza, lo que constituye una debilidad en la prevención de estos trastornos pese a haberse establecido como prioridad en este grupo de edad, tanto en Colombia como en el contexto mundial.

\section{Promoción de la oferta y uso de servicios preventivos en hombres entre 11 y 19 años}

En este grupo de edad los servicios con mayor promoción de la oferta son salud oral (34\%) y consulta médica preventiva (32\%), seguido de la valoración de la agudeza visual (29\%), esto puede estar relacionado con la oportunidad establecida en la norma, de una consulta cada 3 o 4 años dependiendo de la edad. En relación con la consulta médica o psicológica por trastornos relacionados con depresión, ansiedad, intento de suicidio, ésta se promociona en un 10\% y la consejería en uso de drogas, alcohol y tabaco en un $8 \%$, en consecuencia su uso estuvo en 5\% y $10 \%$ respectivamente. Pese a que existe la normatividad que obliga a la implementación de la atención preventiva en salud mental para la población joven, los resultados evidencian que aún no es promocionada por las EAPB, ni es reconocida por la población.

\section{Promoción de la oferta y uso de servicios preventivos en mujeres entre 20 y 29 años}

En este grupo de edad los servicios con mayor promoción de la oferta fueron citología cérvico vaginal (51\%), toma de tensión arterial (40\%), consulta médica preventiva (33\%), y examen de seno y educación para autoexamen (27\%). Llama la atención que en cuatro de seis actividades preventivas exploradas, la utilización sobrepasa la oferta de los servicios. En relación con actividades de detección temprana de VIH y sífilis, la promoción de la oferta obtuvo porcentajes más altos que el uso, siendo sin embargo estos muy bajos: $19 \%$ y $18 \%$, respectivamente.

\section{Promoción de la oferta y uso de servicios preventivos en hombres entre 20 y 29 años}

En este grupo de edad todos los servicios preventivos, promocionados por la EAPB para hombres están por debajo del $30 \%$, los más promocionados fueron consulta médica preventiva (28\%) y toma de tensión arterial (27\%), siendo superior el porcentaje de uso que el de promoción de la oferta. 


\section{Promoción de la oferta y uso de servicios preventivos en mujeres entre 30 y 44 años}

Para este grupo de edad se evidencia mayor promoción de la oferta y uso de algunos servicios preventivos con respecto a los demás grupos de edad, pero en general para todos los servicios evaluados, el porcentaje de uso es superior al porcentaje de promoción de la oferta. Los servicios más promocionados fueron consulta médica $(65 \%)$, citología cérvico vaginal $(62 \%)$, toma de tensión arterial (55\%); mientras los servicios menos promocionados fueron toma de glicemia $(46 \%)$, toma de colesterol total (43\%) y educación en autoexamen de mama (38\%).

\section{Promoción de la oferta y uso de servicios preventivos en hombres entre 30 y 44 años}

En este grupo de edad los servicios más promocionados para hombres fueron consulta médica general (51\%), seguida de la toma de tensión arterial (40\%); el porcentaje tanto de promoción de la oferta como de uso, en el caso de las actividades de detección temprana de cáncer de próstata, son inferiores a 20\%, situación a tener en cuenta, ya que, en Colombia, el cáncer de próstata es la segunda causa de muerte en hombres.

Promoción de la oferta y uso de servicios preventivos en mujeres mayores de 45 añosPromoción de la oferta y uso de servicios preventivos en mujeres mayores de 45 años

En este grupo de edad los servicios más promocionados fueron toma de presión arterial (78\%), citología cérvico vaginal (72\%) y mamografía $(60 \%)$. Se incluyeron pruebas de laboratorio de tamizaje para enfermedad crónica que son obligatorias en la consulta quinquenal del adulto mayor de 45 años (glicemia, creatinina, triglicéridos, uroanálisis, colesterol), estos tuvieron un porcentaje de promoción de oferta superior a $60 \%$, en tanto que las actividades de detección precoz de cáncer de colon no superan el $21 \%$.

Los porcentajes de uso más altos corresponden a toma de presión arterial (85\%), monitoreo de laboratorio como tamizaje o control de enfermedad crónica (>70\%), citología cérvico vaginal (65\%) y mamografía (55\%). El menor porcentaje de uso fue para la colonoscopia $(22 \%)$. Se destaca que mamografía, citología cérvico vaginal y sangre oculta tienen porcentajes de promoción de oferta superiores a su uso. En el resto de actividades indagadas el porcentaje de uso supera al porcentaje de promoción de la oferta. Ver figura 2. 


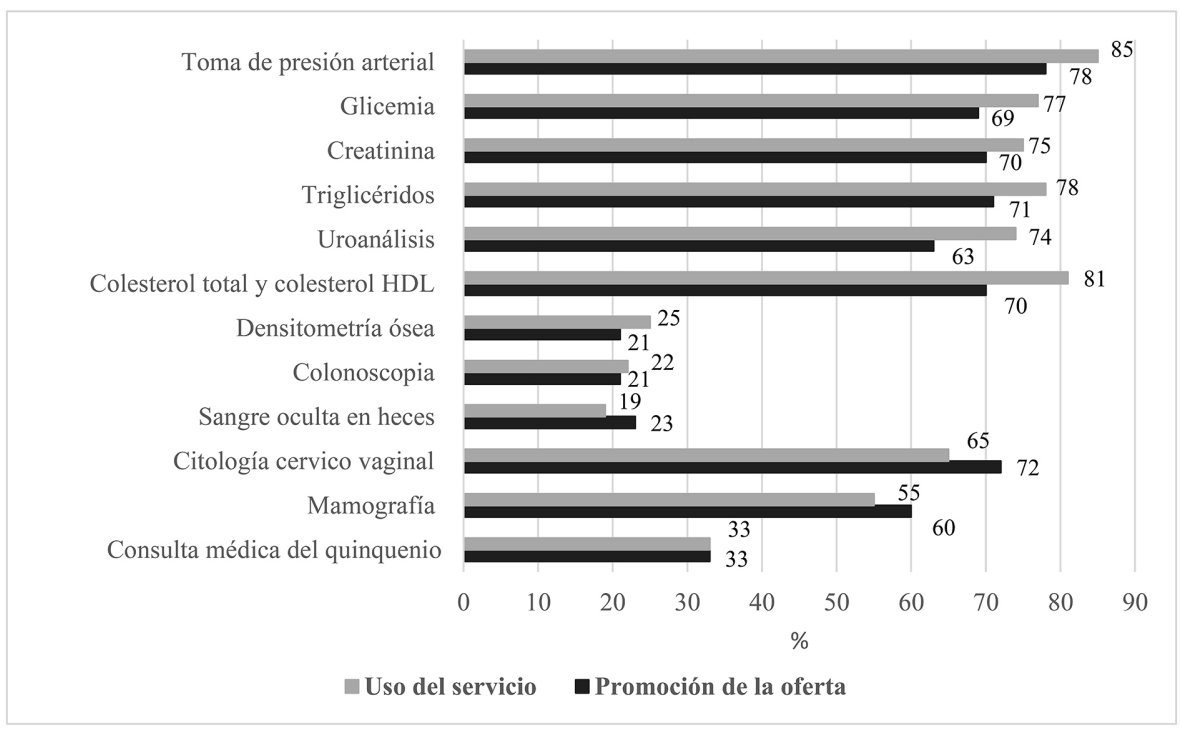

Figura 2. Promoción de la oferta y uso de servicios preventivos en mujeres mayores de 45 años

Fuente: elaboración propia.

Promoción de la oferta y uso de servicios preventivos en hombres mayores de 45 años

Los porcentajes obtenidos en este grupo de edad, tanto en promoción de oferta como en uso, evidencian el énfasis en la atención y detección de los problemas crónicos propios de la edad, arrojando como resultado porcentajes entre $64 \%$ y $83 \%$ para el caso del uso de pruebas de laboratorio, relacionadas con el diagnóstico y control de problemas crónicos y la toma de presión arterial. Sin embargo, en este grupo de actividades los porcentajes de oferta fueron inferiores al uso, encontrándose entre $52 \%$ y $70 \%$.

Los menos promocionados fueron densitometría ósea, sangre oculta en heces, colonoscopia y la consulta médica del quinquenio; en correspondencia, estos servicios fueron los menos usados. Las actividades de detección temprana de cáncer de próstata evidenciaron porcentajes similares de promoción y de uso, siendo el antígeno prostático más usado que el tacto rectal. Ver figura 3. 


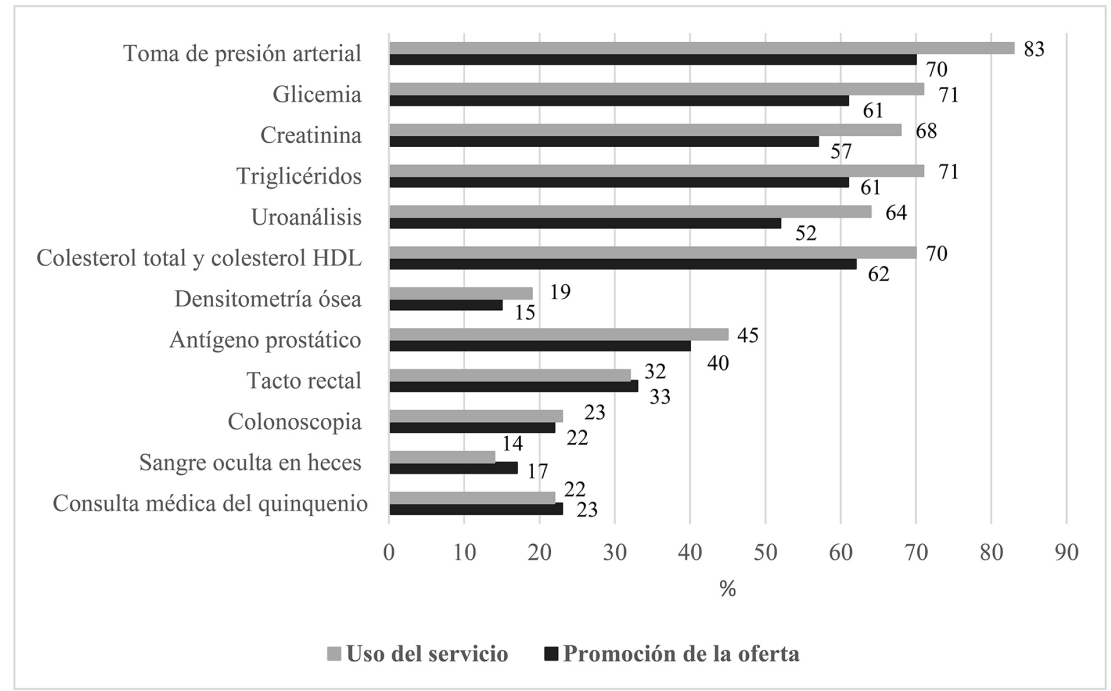

Figura 3. Promoción de la oferta y uso de servicios preventivos en hombres mayores de 45 años Fuente: elaboración propia.

\section{Discusión}

El análisis del acceso efectivo a los servicios de salud desde la percepción de los integrantes de los hogares de la zona urbana del municipio de Palmira evidenció como situación problemática los prolongados tiempos de espera en la atención de urgencias, pero alta resolución de la necesidad demandada y satisfacción por parte de los usuarios. El nivel de satisfacción en este estudio fue mayor (75\%) que el encontrado en el estudio realizado en Cali por Díaz et al. en 2015 , en el cual la proporción de satisfacción por resolución del problema fue de 43,8\% para régimen contributivo y 53,6\% para régimen subsidiado (20).

En el caso de la consulta médica si bien los resultados evidencian alta resolución de la necesidad resalta como problema el tiempo prolongado de asignación de las citas. La continuidad de la atención se ve afectada por el exceso de trámites, la no autorización de servicios y no entrega de medicamentos, lo que coincide con lo documentado en Zarzal, Valle por Gómez et al. (27). Con relación a la consulta especializada, la oportunidad se ve afectada por el tiempo prolongado de autorización del servicio, sumado al tiempo entre la autorización y la atención, en un rango entre 1 día y 6 meses. Hospitalización es un servicio que reportó mejor oportunidad, alta satisfacción por parte de los usuarios (72\%) y alta resolución de la necesidad (86\%); esta última fue, de hecho, mayor que la registrada en Cali en el estudio de Díaz et al. (20): 71,2\% para el régimen contributivo y $38,8 \%$ para el régimen subsidiado.

Los resultados de Palmira se corresponden con los del municipio de Jamundí obtenidos con la aplicación de la misma encuesta, en la cual los servicios de urgencias y hospitalización 
presentaron mejores resultados que la consulta especializada y el acceso a medicamentos. Sin embargo, la percepción sobre la resolución de la necesidad en urgencias fue mayor en Jamundí que en Palmira ( $82 \%$ y 75\%, respectivamente), mientras que en hospitalización fueron similares (85\% y $86 \%$, respectivamente) (25).

Se identificaron dos tipos de situaciones que afectan el acceso a los servicios de salud: situaciones de la oferta y situaciones de la demanda $(28,29)$. Entre las primeras se identifican las que tienen que ver con aspectos organizacionales o institucionales: "muchos trámites para la cita", "no lo atendieron", otras barreras relacionadas con la calidad del servicio como "mal servicio o falta de oportunidad para la atención" y "consultó antes y no le resolvieron el problema"; de oferta de servicios como "el centro de atención no me ofreció el programa". Este trabajo evidenció la escasa promoción de los servicios preventivos por parte de las EAPB a pesar de que la normatividad y las guías de práctica clínica así lo contemplan.

Las tres situaciones que afectan el acceso de la demanda son el gasto de bolsillo: "falta de dinero", la percepción que se tiene sobre la gravedad del problema de salud: "el caso era leve" y el conocimiento acerca del sistema de salud: "no confía en los médicos". Los hallazgos del estudio difieren de los resultados de la Encuesta Nacional de Calidad en Vida (ENCV) de 1997. En cuanto a la razón principal para no acudir a la atención médica al momento de enfermar, la ENCV en 1997 encontró que la razón principal fue "falta de dinero", mientras que en este estudio la principal razón fue "la cantidad de trámites". En la ENCV de 1997 se concluyó que las situaciones que afectan el acceso relacionadas con la demanda representaron el 85,4\%; en este estudio estas representaron el 29\%; lo anterior evidencia que las restricciones del lado de la demanda perdieron importancia en estos últimos 20 años (30).

La revisión de Tovar y Arrivillaga (1) encontró que entre 2000 y 2013 sólo 7 de 31 estudios $(22,6 \%)$ documentaban el acceso en programas de prevención; este estudio evidencia como, a pesar de estar establecidas en el marco de política en salud pública -Plan Decenal de Salud (31), guías de práctica clínica (32) - el enfoque de la atención continúa haciendo más énfasis en lo curativo y menos en la prevención y detección temprana de la enfermedad. La escasa promoción de la oferta de servicios preventivos determinan su poco uso siendo en varios casos incluso mayor el porcentaje de uso que de promoción de la oferta. Esto último sucede especialmente en aquellos servicios preventivos que históricamente han sido promocionados masivamente como son los relacionados con la salud materno infantil (vacunación, valoración del crecimiento y desarrollo del menor de cinco años, entre otros); no así para los servicios de la población adulta en la cual se evidencia desconocimiento y bajo uso. En cambio, los servicios preventivos para la población adulta mayor sí presentan mayores porcentajes de uso evidenciando también el énfasis curativo y/o tardío de la atención preventiva. Estos resultados corroboran lo encontrado en el estudio de Jamundí con relación a que la promoción de la oferta se concentra en los servicios de la primera infancia y va disminuyendo a través de las siguientes etapas del ciclo vital; en correspondencia, el uso también se concentra en los servicios de la infancia, desciende a través de la edad y sufre un incremento en los servicios para mayores de 45 años (25). 
La baja promoción de la oferta por parte de las administradoras de riesgo encontrada corrobora lo documentado por Restrepo-Zea et al. en Medellín (19) como una barrera para acceder a los servicios; la falta de información favorece el no reconocimiento del derecho por parte de los usuarios y conduce al no uso de los servicios, que para el caso de los servicios preventivos se refleja a nivel poblacional en las bajas coberturas de actividades de detección temprana y tratamiento oportuno; y para el caso de los servicios curativos conlleva a la búsqueda de otras alternativas que generan congestión de otros servicios o la búsqueda de soluciones por fuera del sistema.

Se corrobora que en el acceso a los servicios de salud en Palmira prevalece el modelo conceptual de la lógica de oferta y demanda (2) y que el dominio de la accesibilidad se restringe al proceso de buscar y recibir atención, determinado por la disponibilidad y la utilización de los servicios (6). Desde la lógica del modelo de justicia social y derecho a la salud se espera que el servicio sea oportuno, de calidad y sin obstáculos. Los resultados de este estudio evidencian los problemas que encuentran los usuarios en la cotidianidad de los servicios, especialmente en términos de falta de oportunidad, de brechas administrativas que se constituyen en barreras sistemáticas para el acceso, y de percepción de mala calidad, distanciándose de esta manera la atención del objetivo de garantizar el derecho a la salud, más allá del aseguramiento nominal. Lo anterior refuerza lo documentado en el estudio de acceso en población con discapacidad, en el municipio de Zarzal, Valle, cuyos resultados evidenciaron que la condición de estar afiliados a una administradora de riesgos no les garantiza a las personas el acceso efectivo a los servicios de salud; específicamente en los servicios curativos se evidenciaron demoras en la atención, barreras para el acceso a diagnóstico (laboratorios) y tratamiento (medicamentos) (27).

Las mayores barreras para el acceso a los servicios de salud se dan en relación con la oportunidad de la prestación del servicio, la cual se evalúo teniendo en cuenta los tiempos de espera entre la demanda del servicio y la resolución de la necesidad. Con respecto a la continuidad asistencial entendida como "el grado de coherencia y unión de las experiencias en la atención que percibe el paciente a lo largo del tiempo" (33), se evidencia facilidad para acceder a los servicios de consulta médica general y atención inicial de urgencias, pero dificultades en la continuidad hacia servicios más complejos (ayudas diagnósticas, consulta especializada) o para terminación del tratamiento en casa (acceso a medicamentos), impactando negativamente en el goce del derecho a la salud. Para Aller et al. (33) la continuidad asistencial determina una mayor satisfacción de los usuarios, mayor utilización de los servicios preventivos y mejor adherencia a los tratamientos; no obstante, los cambios en los modelos de atención y organización de los servicios de salud así como los avances tecnológicos afectan negativamente dicha continuidad en la medida en que obliga a que los usuarios deban ser atendidos por distintos proveedores en distintas instituciones, lo cual afecta la coordinación de la atención entre niveles.

Por último, se mostró que persisten los problemas que impiden el acceso efectivo a los servicios de salud, lo cual evidencia logros parciales del modelo colombiano en cuanto al objetivo 
de incrementar la equidad en el acceso. Al respecto, la revisión de Tovar y Arrivillaga (1) documenta cómo muchos estudios defienden la tesis de que la implementación de la Ley 100 de 1993 sí mejoró la equidad al ampliar la cobertura, especialmente en la población más pobre. Sin embargo, los estudios en regiones y poblaciones específicas dan cuenta de inequidades en el acceso a los servicios de salud por las diferencias en la atención entre población afiliada y no afiliada, debido a las barreras financieras en la población más pobre-inclusive estando afiliadaquienes además, enfrentan mayor gasto de bolsillo; así como inequidades en el acceso de grupos étnicos minoritarios.

\section{Conclusiones}

Los problemas de acceso relacionados con oportunidad y continuidad asistencial y gasto de bolsillo limitan el ejercicio del derecho a la salud y se distancian de un modelo de acceso basado en la justicia sanitaria, que pone énfasis en el concepto amplio de equidad en salud, incluyendo la equidad en el acceso a servicios de salud de calidad.

Si bien se evidencian problemas en el acceso a la atención médica y de urgencias, resaltan los problemas en el acceso a los servicios preventivos determinados en su mayoría por la escasa promoción de la oferta de los mismos, que a su vez determina el bajo uso. En este contexto la situación más crítica se presenta en la detección temprana de problemas del adulto.

Las propuestas actuales de reforma al modelo de atención para Colombia enfrentan el reto de poner a dialogar un sistema de salud fragmentado y pone énfasis en la curación de la enfermedad, con la atención de las prioridades en salud pública, las cuales deberán fortalecerse desde el abordaje de la promoción de la salud y la prevención de la enfermedad.

Se reconocen como limitaciones del estudio que presenta los resultados descriptivos y no avanza hacia análisis de relaciones del acceso con variables sociales, y además la recolección de información vía autoreporte puede contener sesgo de memoria.

\section{Consideraciones éticas}

El protocolo del estudio fue evaluado y aprobado por el comité de ética de la Facultad de Ciencias de la Salud de la Pontificia Universidad Javeriana, sede Cali.

\section{Contribución de los autores}

El artículo es producto de investigación de una tesis de Maestría en Salud Pública de la Pontificia Universidad Javeriana, Cali, Colombia. Todos los autores contribuyeron en la elaboración del artículo y aprobaron la versión final del manuscrito. 
Clara Inés Sánchez Perafán / Diana Liceth Mora Rojas / Víctor Manuel Botero Henao / Victoria Eugenia Estrada

\section{Financiación}

La recolección de información fue financiada por la Alcaldía Municipal de Palmira, Valle del Cauca, a través de contrato de prestación de servicios con el Centro Nacional de Consultoría.

\section{Conflicto de intereses}

Los autores declaran no tener conflicto de intereses.

\section{Referencias}

1. Tovar LM, Arrivillaga M. Estado del arte de la investigación en acceso a los servicios de salud en Colombia, 2000-2013: revisión sistemática crítica. Rev Geren Polit Salud. 2014;13(27):12-26. http s://doi.org/10.11144/Javeriana.rgyps13-27.eaia

2. Arrivillaga M, Borrero YE. A comprehensive and critical view of conceptual models for access to health services, 1970-2013. Cad Saúde Pública. 2016;32(5):e00111415. https://doi.org/10.1590/0102-311X 00111415

3. Walzer M. Spheres of Justice. New York: Basic Books; 1983.

4. Enthoven A. Health plan: The only practical solution to the soaring cost of medical care reading. Washington DC: Addison-Wesley Publishing Co.; 1980.

5. Hiatt H. America's health in the balance: Choice or chance? New York: Harper \& Row Publishers; 1987.

6. Tanahashi T. Health service coverage and its evaluation. Bull World Health Organ. 1978;56:295-303. h ttps://www.ncbi.nlm.nih.gov/pmc/articles/PMC2395571/

7. Penchansky R, Thomas JW. The concept of access: Definition and relationship to consumer satisfaction. Med Care. 1981;19:127-140. https://www.jstor.org/stable/3764310?seq=1

8. Frenk J. El concepto y medición de la accesibilidad. Salud Pública de México. 1985;27(5):438-453. htt ps://saludpublica.mx/index.php/spm/article/view/422

9. Aday LA, Andersen R. A framework for the study of access to medical care. Health Serv Res. 1974;9:208-220. https://www.ncbi.nlm.nih.gov/pmc/articles/Pmc1071804/

10. Andersen RM, Davidson PL, Ganz PA. Symbiotic relationships of quality of life, health services research and other health research. Qual Life Res. 1994;3:365-371. https://doi.org/ 10.1007/BF00451728

11. Andersen RM. Revisiting the behavioral model and access to medical care: Does it matter? J Health Soc Behav. 1995;36:1-10. https://doi.org/10.2307/2137284

12. Levesque J-F, Harris MF, Russell G. Patient-centred access to health care: Conceptualising access at the interface of health systems and populations. Int J Equity Health. 2013;12:18. https://doi.org/10 $.1186 / 1475-9276-12-18$ 
13. Goddard M, Smith P. Equity of access to health care services: Theory and evidence from the UK. Soc Sci Med. 2001;53:1149-1162. https://doi.org/10.1016/S0277-9536(00)00415-9

14. Braveman P, Gruskin S. Poverty, equity, human rights and health. Bull World Health Organ. 2003;81:539-545. https://www.scielosp.org/article/bwho/2003.v81n7/539-545/

15. Shengelia B, Murray $\mathrm{C}$, Adams $\mathrm{O}$. Beyond access and utilization: defining and measuring health system coverage. In: Murray CJL, Evans DB (eds). Health systems performance assessment. Debates, methods and empiricism. Geneva: World Health Organization; 1976. p.221-234.

16. Vélez M. La salud en Colombia. Pasado, presente y futuro de un sistema en crisis. Bogotá: Penguin Random House Grupo Editorial; 2016.

17. Ministerio de Salud y Protección Social de Colombia. Guías de promoción de la salud y prevención de enfermedades en salud pública. Bogotá; 2007.

18. Céspedes-Londoño JE. Impacto de la reforma del sistema de seguridad social sobre la equidad en los servicios de salud en Colombia. 2002;18(4):1003-1024. https://www.scielosp.org/article/csp/2002.v $18 \mathrm{n} 4 / 1003-1024 / \mathrm{es} /$

19. López ME. Reforma a la salud y reconfiguración de la trayectoria de acceso a los servicios de salud desde la experiencia de los usuarios en Medellín, Colombia. Rev Gerenc Polít Salud. 2011;10(20):97-109. https://www.redalyc.org/articulo.oa?id=54522302007

20. Díaz-Grajales C, Zapata-Bermúdez, Aristizábal-Grisales JC. Acceso y satisfacción con servicios curativos: análisis de casos en afiliados al régimen contributivo y afiliados al régimen subsidiado en un barrio estrato 2 - Cali, Colombia. Access and satisfaction related to curative services: Case analysis of t. 2015;14(29):155-178. https://www.redalyc.org/articulo.oa?id=54543012009

21. Restrepo-Zea J, Silva-Maya C, Andrade-Rivas F, VH-Dover R. Acceso a servicios de salud: análisis de barreras y estrategias en el caso de Medellín Colombia. Rev Gerenc Polit Salud. 2014;13(27):242-265. http://dx.doi.org/10.11144/Javeriana.rgyps13-27.assa

22. Arrivillaga M, Amalfi-Ruiz D, Medina M. Atención en salud de mujeres con lesiones precursoras de cáncer de cuello uterino: evidencia cualitativa de la fragmentación del sistema de salud en Colombia. Rev Gerenc Polit Salud. 2019;18(37). https://doi.org/10.11144/Javeriana.rgps 18-37.asml

23. Domínguez CM, Ramírez SV, Arrivillaga-Quintero M. Acceso a servicios de salud en mujeres transgénero de la ciudad de Cali, Colombia. MedUNAB 2017-2018;20(3):296-309. https://doi.org/ $10.29375 / 01237047.2404$

24. Hurtado LM, Arrivillaga M. Determinación social del acceso a servicios de salud de población infantil en situación de discapacidad. Rev Cubana de Salud Pública, 2018;44(1):100-109. https://www.scie losp.org/article/rcsp/2018.v44n1/100-109/es/

25. Arrivillaga M, Aristizabal JC, Pérez M, Estrada VE. Encuesta de acceso a servicios de salud para hogares colombianos. Gac Sanit. 2016;30(6):415-420. https://doi.org/10.1016/j.gaceta.2016.05.008

26. Secretaria de Salud, Municipio de Palmira. Análisis de situación de salud con el modelo de los determinantes sociales Municipio de Palmira - Valle del Cauca; 2015. 
27. Gómez CA, Pasos LM, González T, Arrivillaga M. Acceso a servicios de salud de personas en situación de discapacidad física en Zarzal (Valle del Cauca, Colombia). Salud, Barranquilla. 2018;34(2):276-283. http://dx.doi.org/10.14482/sun.34.2.61

28. Velandia F, Ardón N, Cárdena J. Oportunidad, satisfacción y razones de no uso de los servicios de salud en Colombia, según la encuesta de calidad de vida del DANE. Colomb Med. 2001;32:4-9. https://co lombiamedica.univalle.edu.co/index.php/comedica/article/view/175

29. López ME. Reforma a la salud y reconfiguración de la trayectoria de acceso a los servicios de salud desde la experiencia de los usuarios en Medellín, Colombia. Rev Gerenc Polít Salud. 2011;10(20):97-109. https://www.redalyc.org/articulo.oa?id=54522302007

30. DANE. Encuesta nacional de calidad de vida 2012. Bogotá; 2013. www.dane.gov.co

31. Ministerio de Salud y Protección Social. Plan Decenal de Salud - PDSP 2012-2021. Bogotá; 2013. https://www.minsalud.gov.co/Documentos\%20y\%20Publicaciones/Plan\%20Decenal\%20-\%2 0Documento\%20en\%20consulta\%20para\%20aprobaci\%C3\%B3n.pdf

32. Ministerio de Salud y Protección Social de Colombia. Guías de Práctica Clínica. Bogotá. http://gpc.m insalud.gov.co/gpc/SitePages/buscador_gpc.aspx.

33. Aller MB, Vargas I, Sánchez I, Henao D. La continuidad asistencial entre niveles percibida por usuarios del sistema de salud en Cataluña. Revista Esp Salud Pública. 2010;84(4):371-387. http://scielo.isciii .es/scielo.php?script $=$ sci_arttext\&pid=S1135-57272010000400003\&lng=es 